\title{
Estrategias de educación en un programa de formación en cirugía pediátrica, como respuesta a la pandemia por COVID-19
}

\author{
Education strategies in a pediatric surgery training program in response \\ to the COVID-19 pandemic
}
Luis Mauricio Figueroa-Gutiérrez ${ }^{1} \mathbb{D}$, Otoniel Franco-Agredo ${ }^{1} \mathbb{D}$, Diego Alfredo Palta-Uribe ${ }^{1}$ (D), Juan Carlos Dueñas-Ramírez ${ }^{\mathbb{D}}$, Jorge Alexander Solarte-Henao² $\mathbb{D}$, Andrés Felipe Marín-Giraldo² $\mathbb{D}$, Marĺa Fernanda Acuña-Saravia' ${ }^{\mathbb{D}}$, Marisol Hinaoui-Salazar $\mathbb{D}^{\text {D, }}$ Guillermo Alberto Sarmiento-Ramírez $\mathbb{D}^{\mathbb{D}}$, José Luis Castillo-Clavijo ${ }^{1} \mathbb{D}$

\footnotetext{
Médico, Especialista en Cirugía Pediátrica, Sección de Cirugía Pediátrica, Universidad del Valle, Cali, Colombia. Médico, residente de Cirugía Pediátrica, Universidad del Valle, Cali, Colombia.
}

\section{Resumen}

Introducción. La pandemia por COVID-I9 ha ocasionado cambios de todo orden en el mundo. Las medidas de emergencia han impactado de manera negativa la oportunidad en la prestación de los servicios de salud y los procesos de educación médica. Los educadores se han visto obligados a innovar y apoyarse mucho más en las tecnologías de la información y la comunicación, con el fin de lograr los objetivos de formación.

Objetivo. Describir la implementación de estrategias de enseñanza para hacer frente a los retos que impone la pandemia por COVID-I9 en un programa de formación en cirugía pediátrica.

Métodos. Revisión de los registros y la asistencia a las actividades académicas programadas antes y después de la llegada del coronavirus a Colombia.

Resultados. Como respuesta a la contingencia, y con el apoyo de las tecnologías de la información y la comunicación, se incrementó el número de actividades académicas en un Ioo \%, se logró duplicar la asistencia a las mismas y la participación de expertos nacionales e internacionales.

Conclusión. La nueva normalidad impuesta por la pandemia por COVID-I9 exige a los educadores en salud desarrollar estrategias de enseñanza y aprendizaje innovadoras las cuales, con la integración de las tecnologías disponibles, permiten enfrentar las dificultades y cumplir con los objetivos de formación en salud.

Palabras clave: COVID-I9; pandemias; educación médica; programas de postgrado; cirugía general; pediatría; telemedicina; proyectos de tecnologías de información y comunicación; tecnología de la información.

Fecha de recibido: 23/09/2020 - Fecha de aceptación: 08/10/2020

Correspondencia: Luis Mauricio Figueroa Gutiérrez, Carrera 19 \# 94-96 Villasol Manzana B casa 19, Pereira, Colombia.

Teléfono: 3137371166.

Dirección electrónica: figueroa.luis@correounivalle.edu.co

Citar como: Figueroa-Gutiérrez LM, Franco-Agredo O, Palta-Uribe DA, Dueñas-Ramírez JC, Solarte-Henao JA, Marín-Giraldo AF, et al. Estrategias de educación en un programa de formación en cirugía pediátrica, como respuesta a la pandemia por COVID-19. Rev Colomb Cir. 2020;35:553-7. https://doi.org/10.30944/20117582.780

Este es un artículo de acceso abierto bajo una Licencia Creative Commons - BY-NC-ND https://creativecommons.org/licenses/by-ncnd/4.0/deed.es 


\begin{abstract}
Introduction. The COVID-I9 pandemic has caused changes of all kinds in the world. The emergency measures have negatively affected the opportunity in the provision of health services and the medical education processes. Educators have been forced to innovate and have supported much more on information and communication technologies, in order to achieve the training objectives.
\end{abstract}

Objective. To describe the implementation of teaching strategies to face the challenges posed by the COVID-I9 pandemic in a pediatric surgery training program.

Methods. Review of records and attendance at scheduled academic activities before and after the arrival of the coronavirus in Colombia.

Results. In response to the contingency, and with the support of information and communication technologies, the number of academic activities was increased by $100 \%$, attendance at them was doubled and the participation of national and international experts.

Conclusion. The new normal imposed by the COVID-I9 pandemic requires health educators to develop innovative teaching and learning strategies, which, with the integration of available technologies, make it possible to face difficulties and meet health-training objectives.

Keywords: COVID-I9; pandemics; education, medical; health postgraduate programs; general surgery; pediatrics; telemedicine; information technologies and communication projects; information technology.

\section{Introducción}

La pandemia por COVID-I9 ha impactado todas las áreas del cuidado de la salud y la educación médica, y los programas de formación en cirugía pediátrica no han sido la excepción. En Colombia, la cancelación de procedimientos de cirugía programada desde el mes de marzo, la suspensión de la consulta ambulatoria presencial, la disminución de los pacientes hospitalizados para priorizar las camas hacia los pacientes afectados por el virus SARS-CoV-2, y los ajustes decretados por el gobierno nacional e implementados por las instituciones de educación superior con el fin de disminuir el riesgo de infección para los residentes y docentes, han afectado negativamente las posibilidades de formación y la adquisición de habilidades, porque ha disminuido ostensiblemente la exposición a los procedimientos que se exigen en una curva de aprendizaje, para que se logren las competencias que se deben alcanzar en esta especialidad $\mathrm{I}$.

Ante esta inédita realidad que plantea la pandemia ${ }^{2}$, los responsables de la educación deben buscar soluciones a las siguientes preguntas: ¿qué hacer con los residentes afectados?, ¿cómo se van a formar? y ¿qué responsabilidad tienen los profesores ante esta situación? '

Para dar respuesta a estos interrogantes, se deben considerar nuevas estrategias que permitan enfrentar esta difícil situación, para que el calendario académico y las prácticas formativas se efecten en el menor grado posible, acercando los profesores y los médicos residentes a los recursos disponibles en Tecnologías de la Información y la Comunicación (TICs), aprovechando los beneficios de la conectividad, la telesalud y la creatividad de todos los miembros del equipo, con el fin de mantener la continuidad del programa mientras se logra la reactivación de los servicios de forma completa ${ }^{3}$.

\section{Métodos}

En el caso del programa de segunda especialidad en Cirugía pediátrica de la Universidad del Valle, para el año 2.020 la planta docente está conformada por 5 profesores vinculados a la institución y 5 cirujanos pediatras asistenciales contratados por el Hospital Universitario del Valle. Todo este equipo se encarga de impartir la docencia a los estudiantes de pregrado de 
medicina y de postgrado de otros programas de especialización como Cirugía general y Pediatría. Para el inicio de la pandemia, estaban matriculados en el programa de segunda especialidad en Cirugía pediátrica dos cirujanos generales nacionales.

Habitualmente, el programa tiene bajo la responsabilidad de los médicos residentes matriculados tres actividades académicas principales por semana, las cuales pueden verse incrementadas de acuerdo con el número de médicos residentes de Cirugía pediátrica y de rotantes adicionales: médicos residentes de Neonatología, médicos residentes extranjeros, estudiantes de medicina de ultimo año en rotación electiva, entre otros. Estas actividades incluyen club de revistas, revisiones de tema, presentación mensual de estadística y complicaciones, y la presentación en Junta de decisiones de los casos complejos, de pacientes hospitalizados o ambulatorios, pendientes de ser llevados a procedimientos.

Para comienzos de este año, por iniciativa de docentes y médicos residentes, se iniciaron las pruebas para la transmisión de las actividades agendadas, con el fin de facilitar y fomentar la asistencia y la participación de más docentes e integrantes de otras especialidades médicas, que por situaciones de horarios en ciertos días no se encontraban en la institución, y definitivamente se decidió utilizar la plataforma $\mathrm{ZOOM}^{\circledR}$.

Debido a la pandemia por COVID-I9, durante marzo, en el momento en que se decretó la suspensión de las actividades académicas grupales y la asistencia de los estudiantes de medicina a las instituciones de salud, se canceló la cirugía programada y la consulta externa, y se disminuyó el número de horas de práctica institucional para proteger a los médicos residentes del contagio, en el programa de segunda especialidad en Cirugía pediátrica de la Universidad del Valle se plantearon modificaciones para incrementar las actividades académicas y el acompañamiento estrecho a los médicos residentes, por medio de tutorías y la utilización de recursos como fotografías y videos.

\section{Resultados}

En el período comprendido entre el I de febrero y el 23 de marzo de 2020 ( 7 semanas), el promedio de asistencia a las 2I actividades académicas programadas fue de I2 personas (entre médicos residentes y docentes, sin contar a los estudiantes de pregrado), de manera presencial o virtual. Posteriormente, durante las primeras 7 semanas de aislamiento, se llevaron a cabo 24 actividades académicas virtuales, con una asistencia promedio de 25 personas (incremento de más del Ioo \%).

Además, mediante la difusión de los enlaces a las reuniones, se pudo convocar a médicos expertos, nacionales e internacionales, con lo que se alcanzó un total de 589 participantes, incluyendo estudiantes de pregrado, médicos residentes y especialistas de Argentina, Brasil, Costa Rica, Ecuador, México, Panamá y República Dominicana.

Esto facilitó compartir experiencias y conocimientos, no sólo con grupos de Cirugía pediátrica sino también de Cirugía de trauma, y con la comunidad global de cirujanos pediatras y urólogos pediatras del Hendren Project (https://www.hendrenproject.org). Una ventaja adicional fue que la grabación de las reuniones y su archivo en la nube permitió tener disponible información científica de calidad, como herramienta de consulta y para la formación de nuevo recurso humano en salud.

\section{Discusión}

Entre las grandes enseñanzas que ha dejado la pandemia por COVID-I9 es que los docentes de los programas de educación médica deben estar dispuestos a cambiar los paradigmas que por décadas han sido parte de la enseñanza y el aprendizaje, poniendo a prueba su ingenio y creatividad, enfrentando con determinación los desafíos de la transición del desarrollo del currículo tradicional a la aplicación de las plataformas en línea ${ }^{4}$. La adopción de esta nueva estrategia se convierte en un reto, tanto para usuarios nuevos como experimentados, ya que implica aprender 
a conocer y familiarizarse con las plataformas de navegación, sus ventajas, desventajas y riesgos ${ }^{4}$.

La División de Educación del American College of Surgeons ha propuesto que, para abordar los desafíos y enfrentar las consecuencias desencadenadas en la formación de los residentes de especialidades quirúrgicas, se pongan a disposición programas de educación quirúrgica en línea, pero lo más importante, que se identifiquen nuevas oportunidades y soluciones creativas que persigan la innovación, no sólo para lograr el mejor entrenamiento en el entorno actual, sino para mantener vigencia para la transformación mediata o futura de la educación ${ }^{5}$.

Dada la incierta duración de la pandemia por COVID-I9, se propone la implementación de estrategias a largo plazo, enfocadas en la modificación de los programas y en la preparación de los médicos residentes para estos tiempos sin precedentes. Una de ellas es la reestruturación de las actividades, con el objetivo de disminuir la exposición buscando que los miembros de los equipos permanezcan sanos ${ }^{6}$. En este orden de ideas, la realización de las revistas médicas o rondas virtuales, en las que todos los miembros del grupo pueden conocer al paciente y sus estudios diagnósticos, pero solo algunos de los miembros los valoran presencialmente, hace parte de una de las acciones establecidas en el servicio.

Otra estrategia tiene que ver con la didáctica, ya que la pandemia ha estimulado la colaboración educativa mundial, incrementando la oferta de recursos en línea, por lo que los programas de especialización medica han hecho la transferencia de sus conferencias presenciales a las plataformas digitales y las han compartido en las redes sociales, con lo que han alcanzado la asistencia de cientos de espectadores ${ }^{6}$.

Dependiendo de cómo se va comportando la pandemia en los diferentes países, y particularmente en las instituciones de salud, se han propuesto protocolos que establecen el funcionamiento de los programas de formación y la actuación de los médicos residentes. Mientras sea posible y no se declare el estado de emergencia, no se deben suspender las actividades de teleducación, como las conferencias virtuales y los clubes de revistas ${ }^{7}$.

Los docentes deben reconocer que la pandemia por COVID-I9 ha creado un entorno de aprendizaje propio y que se está presentado una oportunidad única para que los educadores médicos innoven en sus estrategias de enseñanza, y mientras tanto, los alumnos están experimentando un evento raro en sí mismo, que estimula las partes del cerebro que facilitan el aprendizaje profundo, por lo que la responsabilidad de los docentes está en proporcionar a los estudiantes de pregrado y postgrado una experiencia educativa efectiva ${ }^{8}$. Dentro del programa de segunda especialidad en Cirugía pediátrica de la Universidad del Valle, se ha enfatizado en desarrollar estrategias asincrónicas de aprendizaje con el apoyo de los medios digitales y la inclusión en las presentaciones de más recursos audiovisuales, como videos y fotografías. Probablemente cuando se regrese a la enseñanza presencial, muchos de estos cambios implementados se quedarán definitivamente.

La simulación ha sido usada para el entrenamiento en otras áreas y es fundamental para la adquisición de habilidades, tanto en cirugía abierta como mínimamente invasiva, y mientras algunos equipos no están disponibles para uso en el hogar, existen modelos que se pueden construir a bajo costo para ser usados por los médicos residentes en sus casas ${ }^{9-11}$, lo cual puede ser una oportunidad para considerar durante la pandemia por COVID-I9. El programa de cirugía pediátrica ha sido pionero en este sentido, pero los equipos de simulación sólo estarán disponibles en el momento en el que se autorice el reingreso al laboratorio; mientras tanto se deben aprovechar los equipos ya existentes y desarrollar nuevas ideas y procedimientos que permitan el entrenamiento de los estudiantes de pregrado y los médicos residentes desde el hogar. Igualmente se deben aprovechar estrategias como la grabación de videos didácticos, la virtualización de las clases, técnicas quirúrgicas básicas, semiología quirúrgica y pediátrica, entre otras. 
Como ha sucedido durante toda la historia de la humanidad, en las grandes crisis se originaron desarrollos importantes, por eso, una última propuesta que se podrá considerar a través de las plataformas virtuales es la de implementar un estándar nacional donde los alumnos puedan tener acceso a los profesores más expertos en cada campo, independientemente del programa académico, la ubicación geográfica o la universidad a la que pertenezcan ${ }^{6}$. En este sentido, estos primeros meses han permitido la internacionalización del currrículo a toda Latinoamérica, y a partir del mes de agosto, cumpliendo con uno de los objetivos misionales de la universidad como es la proyección social y la extensión, se estableció un programa de educación virtual en cirugía pediátrica de acceso abierto para todo el personal interesado en estas temáticas.

\section{Consideraciones éticas}

Consentimiento informado. Este estudio es una revisión retrospectiva de registros, que no involucra pacientes, y como tal, no hay necesidad de un consentimiento informado.

Conflictos de interés. Los autores declaran no tener conflictos de interés.

Financiación. Este proyecto no recibió ningún tipo de financiación.

\section{Referencias}

I. Davenport M, Pakarinen MP, Tam P, Laje P, Holcomb GW 3rd. From the editors: The COVID-I9 crisis and its implications for pediatric surgeons. J Pediatr Surg. 2020;55:785-8.

https://doi.org/Io.IoI6/j.jpedsurg.2020.04.009
2. Berwick DM. Choices for the "New Normal". JAMA. 2020;323:2125-6. https://doi.org/Io.IOoI/jama.2020.6949

3. Figueroa LM. Telehealth in Colombia, challenges associated with COVID-I9. Biomédica. 2020;40(Supl 2):pp. https://doi.org/I0.7705/biomedica.5594

4. Rose C, Mott S, Alvarez A, Lin M. Physically distant, educationally connected: Interactive conferencing in the era of COVID-I9. Med Educ. 2020;54:758-9. https://doi.org/IO.IIII/medu.I4192 .

5. Fong ZV, Qadan M, McKinney R, Griggs CL, Shah PC, Buyske J, et al. Practical implications of novel coronavirus COVID-I9 on hospital operations, board certification, and medical education in surgery in the USA. J Gastrointest Surg. 2020;24:1232-6. https://doi.org/I0.I007/sII605-020-04596-5

6. Kwon YS, Tabakin AL, Patel HV, Backstrand JR, Jang TL, Kim IY, Singer EA. Adapting urology residency training in the COVID-I9 era. Urology. 2020;I4I:I5-9. https://doi.org/Io.IoI6/j.urology.2020.04.065

7. Stambough JB, Curtin BM, Gililland JM, Guild GN, Kain MS, Karas V, et al. The past, present, and future of orthopaedic education: Lessons learned from the COVID-I9 pandemic. J Arthroplasty. 2020;35:S60-S64. https://doi.org/IO.IOI6/j.arth.2020.04.032 .

8. Rakowsky S, Flashner BM, Doolin J, Reese Z, Shpilsky J, Yang S, et al. Five questions for residency leadership in the time of COVID-I9: Reflections of chief medical residents from an internal medicine program. Acad Med. 2020;95:II52-4.

https://doi.org/I0.I097/ACM.00000000000034I9

9. Rowley K, Pruthi D, Al-Bayati O, Basler J, Liss MA. Novel use of household items in open and robotic surgical skills resident education. Adv Urol. 2019;2019:5794957. https://doi.org/IO.II55/2019/5794957

Io. Timberlake MD, Garbens A, Schlomer BJ, Kavoussi NL, Kern AJM, Peters CA, Gahan JC. Design and validation of a low-cost, high-fidelity model for robotic pyeloplasty simulation training. J Pediatr Urol. 2020;16:332-9. https://doi.org/IO.IOI6/j.jpurol.2020.02.003

II. Sánchez S, Ariza A. Educación quirúrgica en Colombia en la era del COVID-I9. Rev Colomb Cir. 2020;35:250-5. https://doi.org/I0.30944/201I7582.63I 